\title{
Comparison of natural and forced amplification regimes in plasma-based soft-x-ray lasers seeded by high-order harmonics
}

\author{
Eduardo Oliva, ${ }^{1, *}$ Philippe Zeitoun, ${ }^{1}$ Marta Fajardo, ${ }^{2}$ Guillaume Lambert, ${ }^{1}$ David Ros, ${ }^{3}$ Stephane Sebban, ${ }^{1}$ and Pedro Velarde ${ }^{4}$ \\ ${ }^{1}$ Laboratoire d'Optique Appliquée, ENSTA ParisTech, École Polytechnique ParisTech, CNRS, UMR 7639, F-91761 Palaiseau Cedex, France \\ ${ }^{2}$ GoLP, Instituto de Plasmas e Fusăo Nuclear, Laboratorio Associado, Instituto Superior Técnico, Lisbon, Portugal \\ ${ }^{3}$ Laboratoire de Physique des Gaz et Plasmas, Université Paris Sud 11, Orsay, France \\ ${ }^{4}$ Instituto de Fusión Nuclear, Universidad Politécnica de Madrid, Madrid, Spain
}

\begin{abstract}
The amplification of high-order harmonics $(\mathrm{HOH})$ in a plasma-based amplifier is a multiscale, temporal phenomenon that couples plasma hydrodynamics, atomic processes, and $\mathrm{HOH}$ electromagnetic fields. We use a one-dimensional, time-dependent Maxwell-Bloch code to compare the natural amplification regime and another regime where plasma polarization is constantly forced by the HOH. In this regime, a 10-MW (i.e., 100 times higher than current seeded soft $\mathrm{x}$-ray laser power), 1.5- $\mu \mathrm{J}, 140$-fs pulse free from the parasitic temporal structures appearing on the natural amplification regime can be obtained.
\end{abstract}

PACS number(s): 42.55.Vc, 42.50.Md, 42.65.Ky

Recently, time-resolved $\mathrm{x}$-ray science has undergone a leap forward thanks to the emergence of free-electron lasers (FEL) emitting in soft $\mathrm{x}$ rays $(30 \mathrm{eV}-1 \mathrm{keV})[1,2]$ and hard $\mathrm{x}$ rays (energy greater than $1 \mathrm{keV}$ ) [3]. They provide coherent, microJoules-to-milliJoules energy in hundreds or tens of femtosecond pulses, but in very few, large-scale facilities. The seeding of high-order harmonics (HOH) on plasma amplifiers has been developed in parallel in the soft $\mathrm{x}$-ray range $[4,5]$, delivering several hundred nanoJoules with picosecond pulse duration. This technique allows one to create tabletop sources having optimal spatial properties, as they are fully coherent [5] and aberration free [6]. However, their picosecond duration strongly limits applications in biology, as well as solid-state and plasma physics [7-9]. In addition, previous studies using time-dependent Maxwell-Bloch models $[10,11]$ demonstrated that the temporal structure is actually more complicated than a single amplified $\mathrm{HOH}$ pulse, being temporally multicomponent. The output beam consists of amplified spontaneous emission (ASE), weakly amplified $\mathrm{HOH}$, and a wake field with a complex structure (Rabi oscillations and coherent decay) [10]. In the case of Ni-like Ag pumped under the so-called GRazing Incidence Pumping (GRIP) geometry studied by Al'miev and collaborators, the seed pulse duration (20 fs) was much shorter than the time scale of the atomic response of the lasing ions (few ps) given by electron-ion elastic and nonelastic collisions. As the authors explained, such temporal mismatch leads to the apparition of a long wake of about $6 \mathrm{ps}$ in duration, due to the long-lasting polarization of the plasma by the $\mathrm{HOH}$ pulse. This result might be understood through an analogy with pendulum oscillations. If not excited resonantly, the pendulum reacts on its natural time scale, independently of the applied excitation. Al'miev's work also showed that the ASE extracts a significant part of the energy stored in the plasma. However, the key issue of quantifying the energy of each pulse component (ASE, weakly amplified $\mathrm{HOH}$, and wake) has not been precisely addressed. A more detailed modeling has been done by Kim et al. [12],

\footnotetext{
*eduardo.oliva@ensta-paristech.fr
}

who estimated the energy contained after amplification for low $(1 \mathrm{~nJ})$ and high $(100 \mathrm{~nJ})$ seeding energies. For the second case, the seed is strong enough to saturate the plasma and thus reduces significantly the amplification of both wake and ASE as compared to the low seeding energy case $[10,11]$. However, the output beam is still poorly usable for application since the coherent, short pulse (amplified HOH) contains only $250 \mathrm{~nJ}$ over the total energy of $690 \mathrm{~nJ}$. Previous studies did not provide a solution for extracting most of the energy stored in the plasma in a single hundreds-of-femtoseconds pulse, as required for applications.

In this paper, using the time-dependent Maxwell-Bloch code DEEPONE, we will provide insight into plasma polarization dynamics, presenting the result that the classical regime of seed amplification intrinsically cannot generate the required single-component short pulse, as the system reacts on its own time scale. By continuing with the analogy using the pendulum, we study a solution for resonantly coupling the excitation (here, $\mathrm{HOH}$ ) and the pendulum (here, plasma polarization). We will theoretically describe a regime of forced amplification of the seed pulse that optimizes the adjustment of $\mathrm{HOH}$ properties to plasma hydrodynamic characteristics, leading to a single-component, 140 -fs pulse containing most of the output energy. Due to the different time scales involved in the process, from tens of femtoseconds (HOH pulse duration) to picoseconds (plasma response), Maxwell-Bloch type equations [Eqs. (3) and (5)-(7)] shall be used [11]. The propagation of an electric field is deduced from the following Maxwell wave equation in a plasma:

$$
\Delta \mathbf{E}-\frac{1}{c^{2}} \frac{\partial^{2} \mathbf{E}}{\partial t^{2}}-\frac{\omega_{p}^{2}}{c^{2}} \mathbf{E}=\mu_{0} \mathbf{P},
$$

where $\omega_{p}$ is the plasma frequency. In a one-dimensional model, the transverse Laplacian is neglected, retaining only the derivatives on the $z$ coordinate. The $z$ component of the electric field can be written as $E_{z}=\operatorname{Re}\left[E e^{\left(-i \omega_{0} t+i \kappa z\right)}\right]$, where $\omega_{0}$ is the frequency of the field and $\kappa$ is the wave number. Polarization can be expressed in a similar way. In the slowly varying envelope approximation, we assume that the second derivatives of the envelope function $E(z, t)$ can be neglected (first order in 
the case of polarization). Within this approximation, the wave equation is

$$
\frac{2 i \omega_{0}}{c^{2}}\left(\frac{\partial E}{\partial t}+c \frac{\partial E}{\partial z}\right)=-\frac{\omega_{0}^{2}}{c^{2}}\left(-\frac{\omega_{p}^{2}}{\omega_{0}^{2}} E+\mu_{0} c^{2} P\right) .
$$

With the coordinate change $\tau=t-\frac{z}{c}$, the so-called reduced time (i.e., the referential frame of the photon), and defining $\zeta=c \tau$, we obtain an ordinary differential equation for the propagation of the electric field,

$$
\frac{\partial E}{\partial \zeta}=\frac{i \omega_{0}}{2 c}\left[\mu_{0} c^{2} P-\left(\frac{\omega_{p}}{\omega_{0}}\right)^{2} E\right] .
$$

Equation (3) describes the electric-field propagation through the plasma, amplification by the polarization density, and damping by the free-electron current. The polarization density is given by $\mathbf{P}=\operatorname{Tr}(\rho \mathbf{d})$, where $\rho$ is the density operator and d is the atomic electric dipole. Following [13], the Zeeman coherences are neglected and we only solve, with the same approximations used for the Maxwell equations,

$$
\frac{\partial \rho_{21}}{\partial \tau}=-\gamma \rho_{21}+\frac{i E}{\hbar} z_{21}\left(N_{2}-N_{1}\right) .
$$

In this equation, $\gamma$ is the depolarization rate, which we take to be proportional to the electron-ion collision frequency [14], and $N_{2}, N_{1}$ are, respectively, the population of the upper and lower level of the transition. To obtain the equation for the polarization density, we only have to multiply the equation by the dipole matrix element $z_{21}$ as

$$
\frac{\partial P}{\partial \tau}=\Gamma-\gamma P-\frac{i z_{21}^{2}}{\hbar} E\left(N_{2}-N_{1}\right) .
$$

To model the spontaneous emission, we have added to Eq. (4) a stochastic source term $\Gamma$. This term is a random variable with vanishing correlation function, properly normalized to obtain the expected Lorenzian lasing line profile $[14,15]$. Finally, the populations of the levels involved on the lasing transition are computed using the standard rate equations coupled with the electric field:

$$
\begin{aligned}
& \frac{\partial N_{2}}{\partial \tau}=\sum_{k} C_{k 2} N_{k}+\operatorname{Im}\left(E^{*} P\right) \frac{1}{2 \hbar}, \\
& \frac{\partial N_{1}}{\partial \tau}=\sum_{k} C_{k 1} N_{k}-\operatorname{Im}\left(E^{*} P\right) \frac{1}{2 \hbar} .
\end{aligned}
$$

The populations of the levels involved on the transition are coupled with collisional (de)excitation and radiative deexcitation rates included in the $C_{i j}$ coefficients and with the soft $\mathrm{x}$-ray laser electric field through polarization density [Eqs. (6) and (7)]. We have modeled the $2 p^{5} 3 s J=1 \rightarrow 2 p^{5} 3 p . J=$ 0 transition in Ne-like zinc, situated at $21.2 \mathrm{~nm}$, using a multilevel system. In order to prevent numerical artifacts from generating artificial population oscillations between levels, we implemented other atomic levels as a source or sink of the population. The set of Eqs. (3), (5)-(7) are the MaxwellBloch equations. The temporal resolution of level populations, combined with the stochastic treatment of the ASE, allows one to study several processes required to understand the amplification of $\mathrm{HOH}$ in a plasma, such as Rabi oscillations

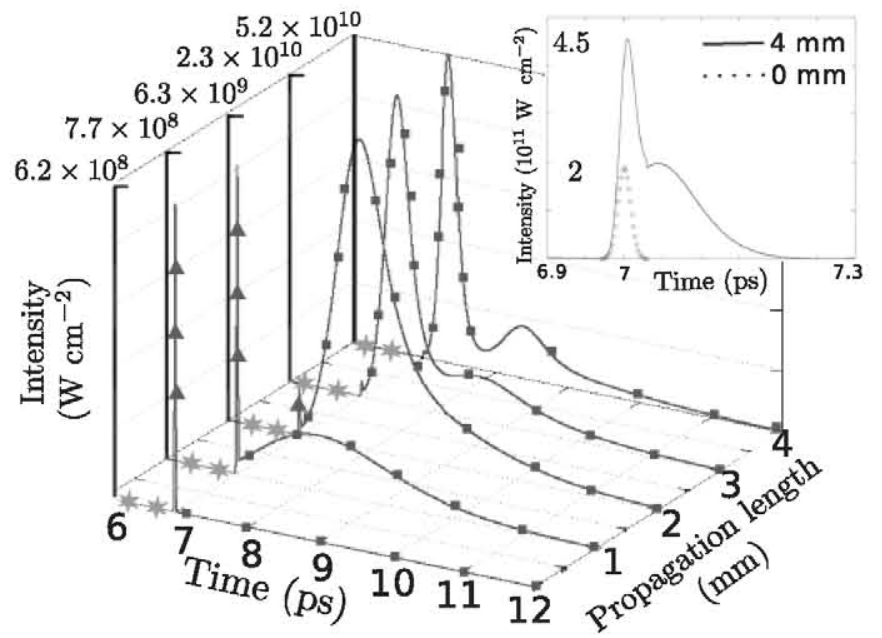

FIG. 1. (Color online) Temporal profile of the intensity of a 20-fs, $50-\mathrm{pJ}$ seeded $\mathrm{HOH}$ beam at different parts of the plasma amplifier with $n_{e}=1.2 \times 10^{20} \mathrm{~cm}^{-3}$ and $T_{e}=550 \mathrm{eV}$. The colors represent the ASE (green stars), HOH (red triangles), and wake (blue squares). Note that intensity is normalized to unity for the sake of visualization. The inset shows the temporal profile of the intensity when the seeding equals $15 \mathrm{~nJ}$.

and amplification of a noisy signal (ASE) mixed with the $\mathrm{HOH}$ signal.

We first modeled the Wang et al. [5] experiment, considering a plasma created by a laser focused on a $4 \mathrm{~mm}$ $\times 30 \mu \mathrm{m}$ focal line, creating a gain cross-sectional area of about $4 \times 10^{-6} \mathrm{~cm}^{2}(36 \times 11 \mu \mathrm{m})$ [16]. The seed pulse is $20 \mathrm{fs}$ full width at half maximum full width at half maximum (FWHM), with $50 \mathrm{pJ}$ of energy. The $\mathrm{HOH}$, which is much more intense than self-emission, immediately polarizes the medium coherently.

This leads to the generation and amplification of a wake that appears from the beginning of the plasma (Fig. 1). The duration of the wake (i.e., duration containing half the energy) increases until it matches the polarization temporal time scale at $z \approx$ $1.5 \mathrm{~mm}$ ( $946 \mathrm{fs}$ ). For longer distances, the interaction between the electric field and atomic populations starts developing Rabi oscillations and saturating the gain, reducing the duration of the wake from $664 \mathrm{fs}$ at $2 \mathrm{~mm}$ down to $360 \mathrm{fs}$ FWHM at $4 \mathrm{~mm}$, as shown in Fig. 1. At this location, the well-known structure existing in a wake having Rabi oscillations and polarization decay [11] is fully developed. After $4 \mathrm{~mm}$, the ASE starts to be strong. The total energy reaches $100 \mathrm{~nJ}$, which is in good agreement with experiments [5]. This energy is completely contained in the wake, whereas the seeded HOH (140 pJ) is mixed with the ASE, which starts to develop $(50 \mathrm{pJ})$. Seeding at higher energy does not solve the problem. The intense $\mathrm{HOH}$ (15 nJ, $20 \mathrm{fs}$ ) not only polarizes the plasma immediately, but also saturates it, reducing the amplification. At $4 \mathrm{~mm}$ (Fig. 1 inset), the $\mathrm{HOH}$ has an energy of only $54 \mathrm{~nJ}$ ( $38 \%$ of the total energy), while the wake reaches $85 \mathrm{~nJ}$. This temporal profile (HOH barely amplified and strong wake) is the signature of a regime in which the $\mathrm{HOH}$ only creates the polarization and then this polarization and the induced electric field evolve independently of the $\mathrm{HOH}$. A detailed study of the creation of the polarization by the $\mathrm{HOH}$ will be useful to 
understand the dynamics of the system. An exact solution of the polarization equation, assuming that population inversion is constant during the time the electric field acts, allows one to determine some characteristic times related to the polarization dynamics. This solution can be factorized on three terms, $P(t)=P_{0} P_{1} P_{2}$, which, for an electric field approximated to a temporal Gaussian distribution, can be written as

$$
\begin{gathered}
P_{0}(t)=-\frac{i z_{21}^{2}}{\hbar} E_{0}\left(N_{2}-N_{1}\right) e^{\frac{\gamma^{2} \sigma^{2}}{2}} \sigma \sqrt{2 \pi}, \\
P_{1}(t)=\frac{1}{\sigma \sqrt{2 \pi}} \int_{-\infty}^{t} e^{-\frac{\left(\tau-t_{\text {cent }}-\gamma \sigma^{2}\right)^{2}}{2 \sigma^{2}}} d \tau, \\
P_{2}(t)=\left\{\begin{array}{cc}
e^{-\gamma\left(t-l_{\text {cent }}\right)} & t \geqslant t_{\text {cent }}, \\
1 & \text { otherwise, }
\end{array}\right.
\end{gathered}
$$

where $E_{0}$ is the maximum seeded electric field, $\sigma$ is the standard deviation of the Gaussian profile of the field, and $t_{\text {cent }}$ is the time of the maximum seed intensity. The numerical values of polarization are given by $P_{0}$, whereas the temporal profile is given by both $P_{1}$ (rising time) and $P_{2}$ (decay time). Polarization rising time has the same characteristic time $\sigma$ as the electric field, and thus follows the seeded pulse with a delay depending on the integral of the $\mathrm{HOH}$ temporal shape. On the other hand, the depolarization characteristic time $\gamma^{-1}$ depends only on plasma properties. In the case of 20 -fs $\mathrm{HOH}$ seeded in a plasma with $n_{e}=1.2 \times 10^{20} \mathrm{~cm}^{-3}$ and $T_{e}=550 \mathrm{eV}$, the two characteristic times are $\sigma=9$ and $\gamma^{-1}=500$ fs.

Thus, depolarization time is more than one order of magnitude greater than rising time. This difference of scale explains the fact that the $\mathrm{HOH}$ itself is not amplified contrary to the wake $[10,11]$. The HOH creates the long-lasting polarization, but due to the intrinsic delay between $\mathrm{HOH}$ and plasma polarization ( 29 fs, i.e., 1.45 times the FWHM), only the final part of the $\mathrm{HOH}$ interacts with the polarized plasma (Fig. 2), preventing an efficient amplification. When polarization reaches its maximum, the $\mathrm{HOH}$ electric field has already dropped to zero. At this moment, the characteristic time scale is no longer given by the seeded beam variance $\sigma$, but only by the plasma properties (i.e., electron-ion collision

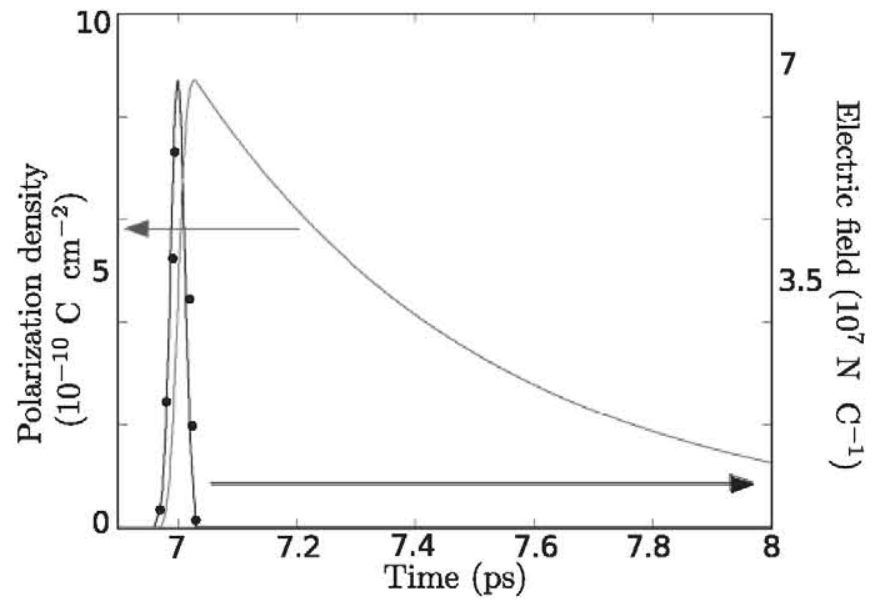

FIG. 2. (Color online) Electric field (black circles) and computed polarization (red continuous line) at $z=0 \mathrm{~mm}$ of a 50-pJ, 20-fs $\mathrm{HOH}$ seeded in a plasma with $n_{e}=1.2 \times 10^{20} \mathrm{~cm}^{-3}$ and $T_{e}=550 \mathrm{eV}$.

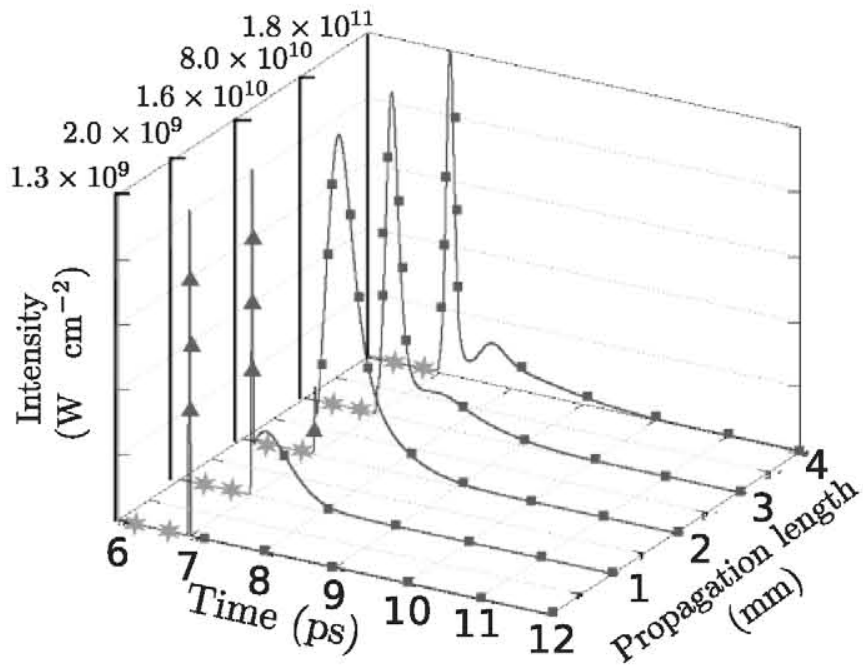

FIG. 3. (Color online) Temporal profile of the intensity of a 20-fs, 1.5-nJ seeded HOH beam at different parts of the plasma amplifier with $n_{e}=2.2 \times 10^{20} \mathrm{~cm}^{-3}$ and $T_{e}=531 \mathrm{eV}$. The colors represent the ASE (green stars), $\mathrm{HOH}$ (red triangles), and wake (blue squares). Note that intensity is normalized to unity for the sake of visualization.

time). This time is about $500 \mathrm{fs}$ and thus polarization decays exponentially during picoseconds, as shown in Fig. 2. This long polarization induced by the $\mathrm{HOH}$ will create and amplify the wake. Consequently, if the seed $\mathrm{HOH}$ temporal profile matches the polarization temporal profile, all the $\mathrm{HOH}$ will be amplified.

An efficient way to enhance this matching consists of increasing the electron density. The electron-ion collision frequency $v_{e i}$ is higher, and thus the polarization decay time [17] is shorter. As we showed in a previous study, a simple way to increase the density consists of making wider plasmas, thereby enhancing at the same time the gain and the maximum energy that can be extracted $[16,18]$. With this consideration in mind, we have studied a plasma created by focusing a laser on a $4 \mathrm{~mm} \times 100 \mu \mathrm{m}$ focal line, leading to an electron density of $n_{e}=2.2 \times 10^{20} \mathrm{~cm}^{-3}$ and electron temperature of $531 \mathrm{eV}$. The gain cross-sectional area is about $6 \times 10^{-5} \mathrm{~cm}^{-2}$ $(120 \times 51 \mu \mathrm{m})$ [18]. Figure 3 shows the resulting intensity when seeding this plasma with a $1.5-\mathrm{nJ}$ beam with a duration of $20 \mathrm{fs}$.

As expected, increasing the electron density has shortened the duration of the pulse, but, nevertheless, the dynamic is similar to the former case: the seeded HOH is barely amplified and creates a long wake, which carries all the energy of the pulse. The shortening of the wake and the weak amplification of the seeded $\mathrm{HOH}$ are the results of the polarization dynamic. Figure 4 shows the polarization density and the seeded electric field. As the electron density is higher, the maximum value is now three times greater than in the previous case, and the depolarization time is now reduced to $\gamma=176 \mathrm{fs}$. The resulting wake is shorter than in the previous case. Nevertheless, this time is still an order of magnitude greater than the seed duration, explaining both that the $\mathrm{HOH}$ is barely amplified and also the creation of the wake.

Then, in order to amplify efficiently the $\mathrm{HOH}$ itself, it is necessary to increase the electron density of the plasma and the 


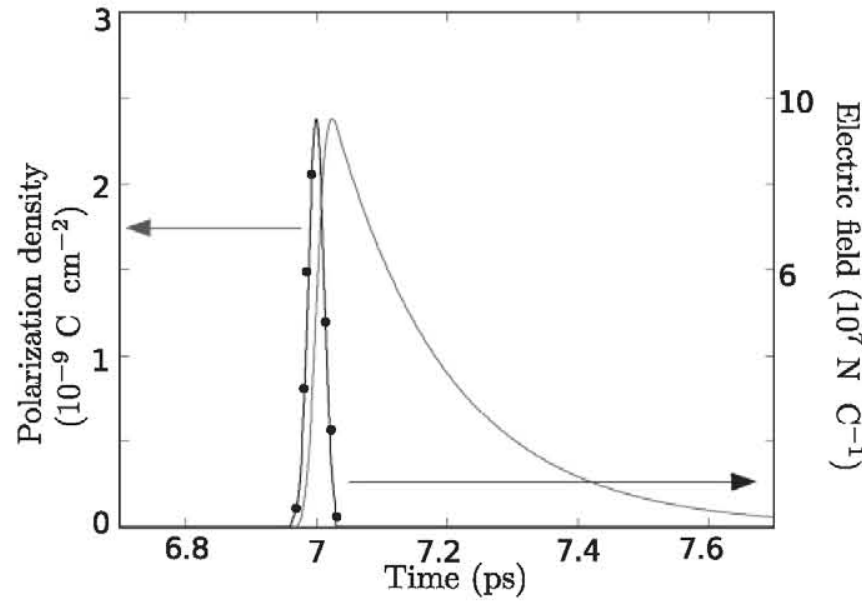

FIG. 4. (Color online) Electric field (black circles) and computed polarization (red continuous line) at $z=0 \mathrm{~mm}$ of a $1.5-\mathrm{nJ}, 20-\mathrm{fs} \mathrm{HOH}$ seeded in a plasma with $n_{e}=2.2 \times 10^{20} \mathrm{~cm}^{-3}$ and $T_{e}=531 \mathrm{eV}$.

duration of the seeded beam to better match the depolarization time. It is worth mentioning that increasing the duration of the $\mathrm{HOH}$ is not only interesting to transfer the energy from the wake to the $\mathrm{HOH}$, but also to enhance polarization, as shown in Eq. (8). Figure 5 depicts the polarization density at $0 \mathrm{~mm}$ (red) and the electric field of a 200-fs (ten times larger), 1.5-nJ HOH (black).

As usual, polarization rises following the integral of the electric field. The delay between the electric field and polarization reduces the amplification of the first half of the $\mathrm{HOH}$. Nevertheless, the second half of the $\mathrm{HOH}$ is efficiently amplified. This is due to the fact that the delay between the maxima of the electric field and polarization, although high in this case ( $110 \mathrm{fs}$ ), is only 0.55 times the FWHM of the seeded beam, allowing an efficient coupling between the $\mathrm{HOH}$ and polarization. This is clearly demonstrated in Fig. 6, showing the spatiotemporal amplification of a 200-fs, $1.5-\mathrm{nJ} \mathrm{HOH}$ pulse.

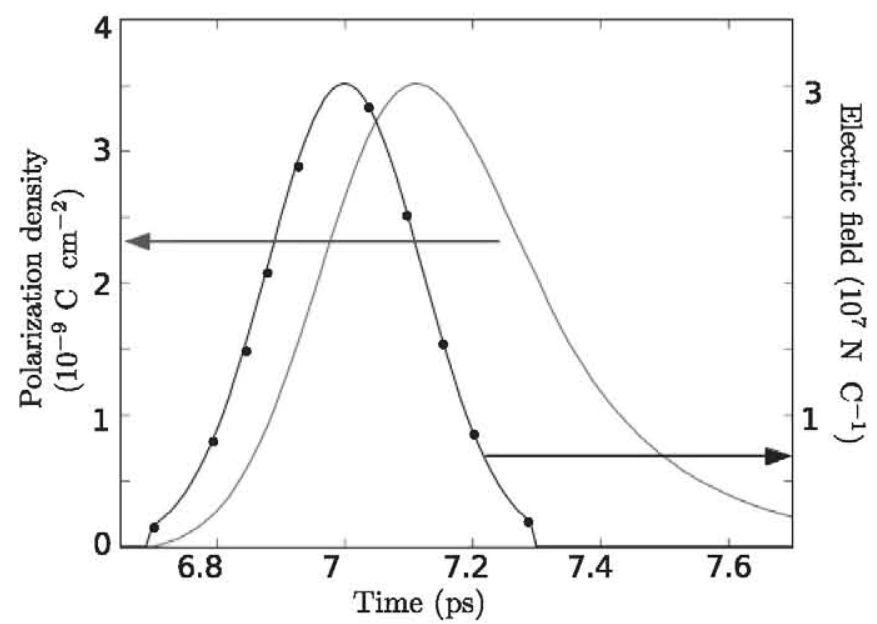

FIG. 5. (Color online) Electric field (black circles) and computed polarization (red continuous line) at $z=0 \mathrm{~mm}$ of a $1.5-\mathrm{nJ}, 200-\mathrm{fs}$ $\mathrm{HOH}$ seeded in a plasma with $n_{e}=2.2 \times 10^{20} \mathrm{~cm}^{-3}$ and $T_{e}=531 \mathrm{eV}$.

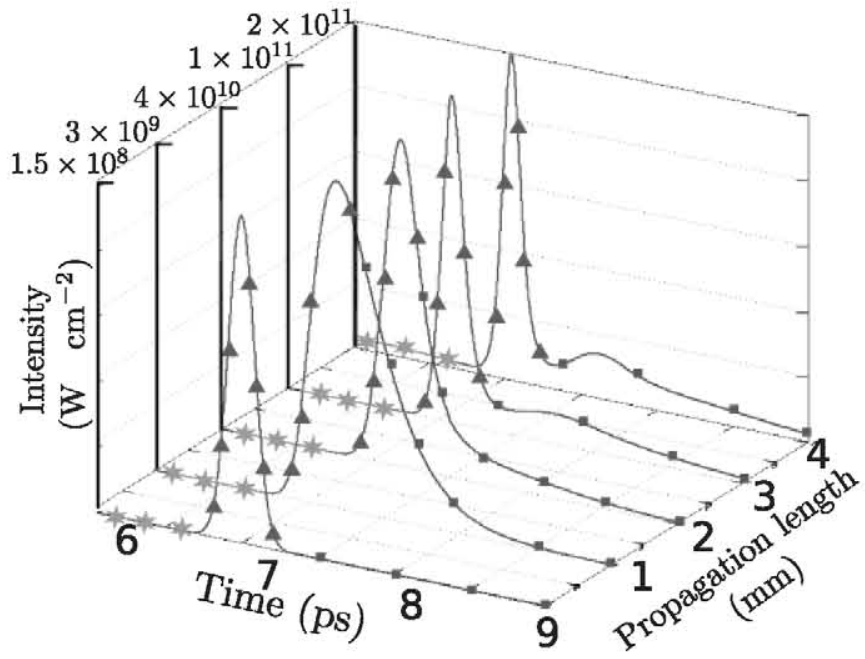

FIG. 6. (Color online) Temporal profile of the intensity of a 200fs, 1.5-nJ seeded $\mathrm{HOH}$ beam at different parts of the plasma amplifier. The colors represent the ASE (green stars), HOH (red triangles), and wake (blue squares). Note that intensity is normalized to unity for the sake of visualization.

The amplification dynamics is completely different from previous cases. The HOH is directly coupled with the polarization from the beginning of the plasma, forcing the amplification of the $\mathrm{HOH}$ itself. In this forced amplification regime, the $\mathrm{HOH}$ fits polarization earlier in the plasma $(z \approx 1 \mathrm{~mm})$ with a duration of $340 \mathrm{fs}$. From that moment, saturation starts to reduce the duration of the pulse ( $260 \mathrm{fs}$ at $z=2 \mathrm{~mm}$ ), and, as in the 20-fs case, drives Rabi oscillations at $4 \mathrm{~mm}$. Due to the higher area of the amplifier, ASE appears for shorter distances between 3 and $4 \mathrm{~mm}$. Nevertheless, between 2 and $3 \mathrm{~mm}$, the amplified beam exhibits the best properties, with a duration of about $240 \mathrm{fs}$ and an energy of about $1 \mu \mathrm{J}$ on a total of $1.5 \mu \mathrm{J}$, i.e., $67 \%$ of the energy is contained into the $\mathrm{HOH}$ itself.

In Table I, the characteristics of the beam at the optimal location in the plasma $(2.5 \mathrm{~mm})$ for natural and forced amplification and low and high seeding energy are shown. As explained, in the natural amplification regime (20-fs seed), the energy is mainly contained in the wake $(99.8 \%$ in the 50 -pJ case, $59 \%$ in the $15-\mathrm{nJ}$ case). Pulse duration is given by saturation effects and depolarization time $\gamma$, leading to about 500 fs for a fully developed wake, as in the $50-\mathrm{pJ}$ case. The $15-\mathrm{nJ}$ case does not completely develop the wake due to the strong saturation of the medium by the $\mathrm{HOH}$ (as the low amplification suggests). On the other hand, in the forced amplification regime, energy is efficiently transferred to the $\mathrm{HOH}$ ( $67 \%$ for 1.5 -nJ seed, $88 \%$ for 15 -nJ seed), halving the duration of the pulse compared to the natural regime. The amplification factor of the $\mathrm{HOH}$ is also increased by more than two orders of magnitude. In this case, the output beam consists of a coherent, strongly amplified $\mathrm{HOH}$ pulse free of temporal structure.

In conclusion, we have developed the one-dimensional, time-dependent Maxwell-Bloch code DEEPONE to study the amplification of $\mathrm{HOH}$ in a plasma amplifier. Using this code, we have identified another amplification regime, called the 
TABLE I. Total energy, energy contained in the HOH, percentage of the total energy contained in the harmonic, duration of the amplified pulse (i.e., duration containing half the energy), and amplification of the $\mathrm{HOH}$ and wake as a function of seed pulse duration and energy at $z=2.5 \mathrm{~mm}$.

\begin{tabular}{|c|c|c|c|c|c|c|c|c|}
\hline focal width $(\mu \mathrm{m})$ & $\tau_{\text {seed }}(\mathrm{fs})$ & $E_{\text {seed }}(\mathrm{nJ})$ & $E_{\text {tot }}(\mathrm{nJ})$ & $E_{\mathrm{HOH}}(\mathrm{nJ})$ & $E_{\mathrm{HOH}}(\%)$ & $\tau_{\mathrm{ampl}}(\mathrm{fs})$ & $\frac{E_{\mathrm{HOH}}}{E_{\text {seed }}}$ & $\frac{E_{\text {wake }}}{E_{\text {seed }}}$ \\
\hline 30 & 20 & 0.05 & 46 & 0.1 & 0.2 & 528 & 2 & 918 \\
\hline 30 & 20 & 15 & 83 & 34 & 41 & 418 & 2.3 & 3.3 \\
\hline 100 & 20 & 1.5 & 1100 & 10 & 0.01 & 260 & 6.7 & 727 \\
\hline 100 & 200 & 1.5 & 1500 & 1000 & 67 & 240 & 666 & 333 \\
\hline 100 & 200 & 15 & 1700 & 1500 & 88 & 140 & 100 & 13 \\
\hline
\end{tabular}

forced amplification regime. The transition between the previously studied natural amplification regime and this regime depends on the adjustment of seed $\mathrm{HOH}$ properties to plasma parameters. The increase of the duration of the seeded pulse and reduction of the characteristic depolarization time of the amplifier (by increasing electron density) favors the forced amplification regime, where the HOH is fully coupled with the polarization, ensuring an efficient amplification. Within $2.5 \mathrm{~mm}$ of plasma, a short (140-fs), single-component pulse with energy of $1.5 \mu \mathrm{J}$ is obtained, keeping ASE negligible.
The 10-MW power attained by this source is 100 times greater than the experimental values using a solid target $[\%$, and its spatial and temporal coherences makes it suitable for many applications.

The authors would like to acknowledge the financial support provided by the LASERLAB II (SFINX) European Project No. 228334 and RTRA "Triangle de la Physique" project (SHYLAX).
[1] V. Ayvazyan et al., Eur. Phys. J. D 37, 297 (2006).

[2] T. Shintake et al., Nature Photon. 2, 555 (2008).

[3] P. Emma et al., Nature Photon. 4, 641 (2010).

[4] P. Zeitoun et al., Nature (London) 431, 426 (2004).

[5] Y. Wang, E. Granados, F. Pedaci, D. Alessi, B. M. Luther, M. Berrill, and J. J. Rocca, Nature Photon. 2, 94 (2008).

[6] J. P. Goddet et al., Opt. Lett. 34, 2438 (2009).

[7] H. N. Chapman et al., Nature (London) 448, 676 (2007).

[8] B. Nagler et al., Nature Phys. 5, 693 (2009).

[9] L. Young et al., Nature (London) 466, 56 (2010).

[10] I. R. Al'miev, O. Larroche, D. Benredjem, J. Dubau, S. Kazamias, C. Möller, and A. Klisnick, Phys. Rev. Lett. 99, 123902 (2007).

[11] C. M. Kim, J. Lee, and K. A. Janulewicz, Phys. Rev. Lett. 104, 053901 (2010).
[12] C. M. Kim, K. A. Janulewicz, H. T. Kim, and J. Lee, Phys. Rev. A 80, 053811 (2009).

[13] A. Sureau and P. B. Holden, Phys. Rev. A 52, 3110 (1995).

[14] O. Larroche, D. Ros, A. Klisnick, A. Sureau, C. Möller, and H. Guennou, Phys. Rev. A 62, 043815 (2000).

[15] S. Chandrasekhar, Rev. Mod. Phys. 15, 1 (1943).

[16] E. Oliva, P. Zeitoun, S. Sebban, M. Fajardo, P. Velarde, K. Cassou, and D. Ros, Opt. Lett. 34, 2640 (2009).

[17] D. S. Whittaker, M. Fajardo, P. Zeitoun, J. Gautier, E. Oliva, S. Sebban, and P. Velarde, Phys. Rev. A 81, 043836 (2010).

[18] E. Oliva, P. Zeitoun, P. Velarde, M. Fajardo, K. Cassou, D. Ros, S. Sebban, D. Portillo, and S. le Pape, Phys. Rev. E 82, 056408 (2010). 